\title{
Use of the CANopen protocol in vibration control systems of rotary machines
}

\author{
Oleg Kovalyov, and Yurii Chebakov* \\ Dmitrov Fisheries Technological Institute (branch) of the Astrakhan State Technical University; \\ Dmitrov, Russia
}

\begin{abstract}
Currently, most accidents and disasters at power plants are associated with fatigue failure of machines, mechanisms and assemblies caused by vibration. To prevent real threats of man-made disasters, there is an urgent need for objective monitoring of the current technical condition of mechanisms without disassembling them, during operation, repair and adjustment. The article proposes to use innovative approaches in the design of reliable and high-speed communication systems for the control of diagnostics and vibration monitoring of rotary-type machines in the form of a high-level network protocol running on top of the physical interface CAN (Controller Area Network). The aim of the study is to develop proposals for the use of the CANopen protocol in vibration control systems for rotary-type machines. The authors of the article analyzed the causes of man-made accidents at energy enterprises, identified the goals and objectives of vibration control of rotary machines and substantiated the possibility of using the CANopen protocol in vibration control systems. This will allow the exchange of data between devices from different manufacturers and ensures the interchangeability of devices. Thus, the authors concluded that the use of the CANopen protocol in vibration control systems for rotary-type machines is a promising direction that allows engineers to solve the necessary technical problems using modern methods.
\end{abstract}

\section{Introduction}

Most accidents and disasters at power plants are associated with vibration-induced fatigue failure.

The most serious accident at the Kashirskaya SDPP in 2002 at the turbine generator at st. No. 3 with a steam turbine K-300-240 and a TVM-300 generator. At the same time, a steam turbine, a condenser, an electric generator were completely destroyed, the foundations and supporting columns were damaged, a fire broke out, and the roof of the turbine hall collapsed. The cause of the accident was high longitudinal vibration and torsional vibrations

\footnotetext{
${ }^{*}$ Corresponding author: chebakovut@gmail.com
} 
as a result of the destruction of the generator sleeve and a short circuit in the stator circuit [1].

On 17/08/2009, an accident occurred at the Sayano-Shushenskaya HPP. 75 people died, 13 were injured. Material damage from the accident exceeded RUR $40 \mathrm{bln}$ [2]. The cause of the accident is high revolving vibration with an amplitude of $800 \mu \mathrm{m}$ (instead of $100 \mu \mathrm{m}$ at the norm) as a result of defects in the impeller and imbalance of the rotor left after installation and overhaul [1].

As known, vibration is not a parameter, such as temperature. Vibration is a signal, or rather the sum of signals from various elements and assemblies of a technical product. And to isolate informative components from the vibration signal, and even more so to establish strict objective norms, is a very, very difficult task.

It has been established that a significant part of the failures of mechanical engineering products is associated with fatigue breakdowns of parts under the action of alternating stresses during vibrations. To prevent vibration breakdowns, it is necessary to know the natural frequencies and vibration modes of parts [3].

When developing technical specifications, it becomes necessary to design a reliable and high-speed communication system for process control. The difficulty in choosing a network and protocols lies in the selection of all the characteristics and requirements of the network and the configuration of the nodes. In this case, it is possible to use the universal CANopen protocol based on CAN buses.

CANopen is designed as a high-level network protocol that runs on top of the physical interface CAN (Controller Area Network), which allows data exchange between devices from different manufacturers, and ensures device interchangeability. The CANopen protocol is used mainly in motion control systems, in assembly, welding and transport units.

\section{Study methodology}

Vibration is mechanical vibrations of the body about the equilibrium position [4]. Vibration is one of the most important diagnostic signs and can be used to monitor the current technical condition of mechanisms without disassembling them, during manufacture, operation, repair and adjustment.

For rotary-type machines, the main suppliers of diagnostic information characterizing the current technical condition are information signals from sensors about the vibration of the rotor supports and the rotor itself. By analyzing the nature of vibration, one can get the information of interest for almost every defect in installation, manufacturing or wear.

Based on this analysis, the following main tasks are solved:

monitoring - recognition of the current technical state of the mechanism;

diagnostics - detecting the causes and conditions that cause malfunctions, and then making informed decisions to eliminate them.

The main task of monitoring is to control the general technical condition of the device and correctly detect emergency situations. Most often, monitoring systems are connected to emergency protection devices that turn them off in the event of an emergency.

The rapid development of microprocessor technology and technology contributed to the development of diagnostic methods and tools. The tools that are most often used for vibration monitoring usually perform tasks for operational adjustment during operation and repair and make it possible to solve the following tasks:

- reasonably determine the timing of repair and commissioning work, monitor their implementation;

- monitor the current technical condition of mechanisms

- $\quad$ - prevent "sudden" breakdowns that entail production interruption; 
- $\quad$ reduce the financial costs of operating the equipment;

- improve the quality of production and staff qualifications;

- $\quad$ reduce the need for spare parts, materials and equipment;

- $\quad$ extend the overhaul period and the service life of the equipment.

The turbine unit vibration control system (VCS) is designed to collect, archive, display on a computer screen and transfer to a higher level of the APCS current and accumulated information about the vibration state of turbine units (TU) at thermal power plants (TPPs) of the energy complex of Russia. With the appropriate setting, the VCS equipment generates control actions in the alarm systems and emergency shutdown of the TU.

The block diagram of one of the options for the implementation of VCS [5, 6] is shown in Figure 1. The VCS consists of a system controller and several subsystems interconnected by a CANopen interconnect interface. The system ensures the functioning of each module separately, as well as the joint operation of modules in any combination. Each of the modules is used only when it is necessary to perform the corresponding functions, and does not have to be included in the system.

The data archiving and visualization module is designed to receive measurement results and calculations from the inter-block interface of the system, archive them, display and transfer them to the next level of the APCS.

The module is made on the basis of a top-level controller, which is used as a personal computer

The vibration measurement module of the supports is designed to control the vibration parameters of the supports by receiving and preprocessing data from intelligent vibration acceleration sensors (IS), transmitting the processed data via a digital communication line to the control interface, indicating the measured values [7]. Vibration Acceleration ISs include multiple piezo accelerometers and an Intelligent Vibration Acceleration Sensor (IVAC) controller.

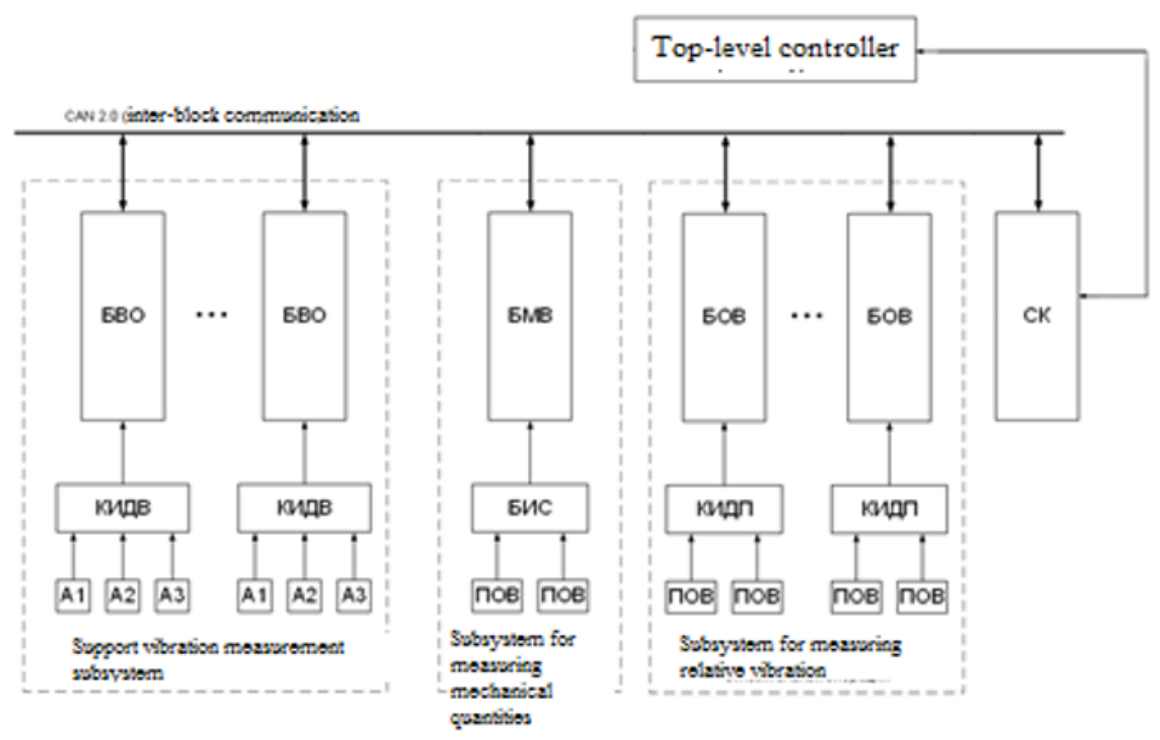

Fig. 1. Structural diagram of the vibration control system.

The module for measuring the relative vibration displacement of the shaft is designed to measure the vibration displacement of the rotating shafts inside the bearings and measure the gap between the sensor and the shaft by receiving and preprocessing the data coming from 
the gap IS, transmitting the processed data via a digital communication line to the control panel, indicating the measured and calculated parameters and generating output analog and discrete signals in order to connect additional recording and executive equipment [4]. The ID of the gap includes up to two transducers of the relative vibration RVT and the IS controller of vibration displacement (VDISC).

The module for measuring mechanical quantities is designed to measure the displacement and linear expansion of the rotor by receiving and preprocessing data coming from the gap IS, transmitting the processed data via a digital communication line to the control panel, indicating measured and calculated parameters and generating output analog and discrete signals for the purpose of connecting additional recording and executive equipment [8]. The subsystem includes the following blocks:

- relative vibration transducer (RVT) - provides the formation of a unified output signal 0 ... $10 \mathrm{~V}$, proportional to the current value of the gap between the sensor and the shaft;

- displacement measuring unit (DMU) - provides the conversion of the unified output signal of the RVT into digital form, its preliminary processing (filtering, linearization, compensation of temperature dependence) and transmission via the RS-485 interface to the middle level of the subsystem;

- mechanical value unit (MVU) - provides data receipt from one or several DMUs, their indication and intermediate archiving, as well as transmission to other subsystems using the inter-block interface CAN [5]. Like other units, MVU provides the formation of output analog and discrete signals for connecting additional recording equipment.

The system controller (SC) provides the following functions:

- - coordination of the interaction of other subsystems and blocks that make up the VCS;

- $\quad$ - coordination of CAN-Ethernet interfaces in order to connect the upper-level controller to the system.

Remote input-output unit (RIOU) - provides input and conversion into digital form of unified signals (current, voltage) or signals from thermocouples and thermocouples, with subsequent transmission of the obtained values to the middle level of the system via the RS-485 interface. The subsystem allows the use of both proprietary RIOU and ready-made input-output units that provide interaction via the MODBUS RTU protocol in the slave mode;

Input-output unit (IOU) - provides data receipt from one or more RIOU, as well as input, conversion into digital form and preprocessing of unified signals, their indication, intermediate archiving and transmission to other subsystems using the CAN inter-block interface via the CANbus bus. Like other blocks of the middle level, IOU provides the formation of output analog and discrete signals for connecting additional recording equipment.

\section{Study results}

CANopen is an open, high-level network protocol for connecting embedded devices in onboard transport and industrial networks. The authors propose to use the CAN real-time protocol as a network and transport layer. The CANopen standard consists of an addressing scheme, several small communication protocols and an application layer defined by the device profile. Communication protocols support network management, device monitoring, and communication between nodes, including a simple transport layer for message segmentation / desegmentation $[9,10]$.

CANopen is a CAN based upper layer protocol. The protocol is designed for motionoriented machine control networks such as data processing and debugging systems. CANopen services, protocols, and network objects include the following items:

Device Model and Object Dictionary (OD) 
Process Data Objects (PDO)

Service Data Objects (SDO)

Network Management Objects (NMT) Time)

Special function objects for synchronization, alarm and timing functions (Sync, Emcy,

Error control services

CANopen frees the developer from having to deal with CAN-related details.

The proposed device model for use is structured, and, as shown in Figure 2, consists of the following main levels:

Application layer of the application.

Network block and communication lines.

Object Dictionary

Application layer of the application This layer is a system of settings and data exchange in real time, as well as mechanisms for interfacing devices and nodes of the CANopen network. Application level functionality allows to logically divide applications into separate service objects in this level [11]. The service object provides the main functionality of the entire protocol, which includes the services associated with this protocol.

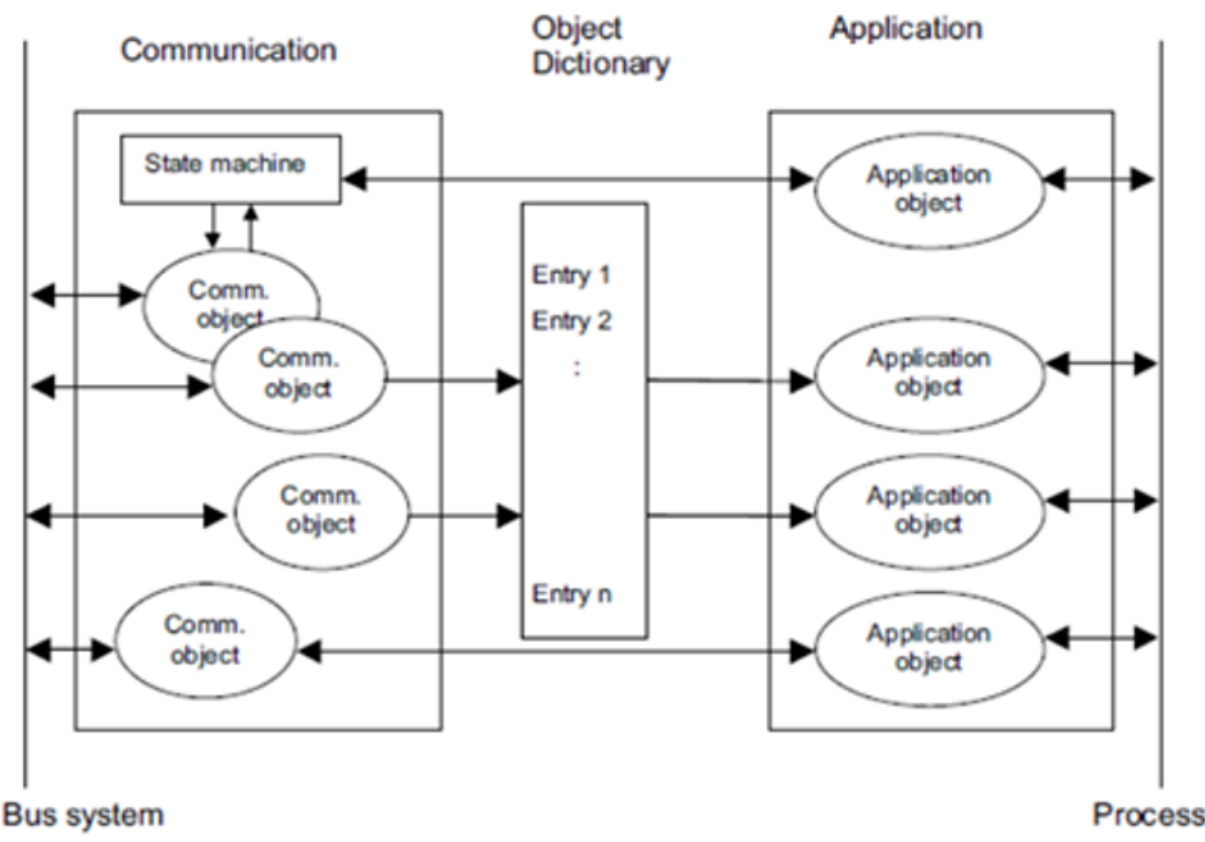

Fig. 2. CANopen device model

Applications interact by invoking the services of a service object at the application layer. To enable these services, this object exchanges data via the CAN Network with the service object(s) of another node using the specified protocol [10].

Object Dictionary is a two-level list or table with objects.

The first level of the table is numbered with indices from 0 to 65535 (16 bits). The second level of the table is numbered with subindices from 0 to 255 ( 8 bits). The items in this table contain data exchanged between nodes in the CANOpen network (an example is shown in Figure 3). 
Each CANopen communication node must have its own object dictionary. Typically, manufacturers of devices that have the ability to connect via the CANOpen protocol compile object dictionaries in EDS format.

\begin{tabular}{|l|l|l|l|l|l|}
\hline $\begin{array}{l}\text { Index } \\
\text { (hex) }\end{array}$ & $\begin{array}{l}\text { Object } \\
\text { (Symbolic Name) }\end{array}$ & Name & Type & Attrib. & M/O \\
\hline
\end{tabular}

Fig. 3. Object dictionary structure

Index: the Index column indicates the position of objects in the object dictionary. This acts as an address to reference the desired data field.

A subindex is used to refer to data fields in a complex object such as an array or record.

Object - contains the name of the object and is used to indicate what type of object is at a particular index in the object dictionary.

Name - provides a simple textual description of the function of a particular object.

Type gives information about the type of an object. For example: boolean, floating point, unsigned integer, signed integer

Attribute defines the access rights for a specific object. For example: rw (read-only), wo (write-only), ro (read-only), Const (read-only and value is a constant number).

$\mathrm{M} / \mathrm{O}$ determines whether an object is required or optional.

To index data, one needs two parameters - an index and a subindex (if the data is used in a complex object such as an array). The cells of this table can contain absolutely any data, for example: current values of analog or discrete inputs, control values for output signals, settings, information about the device and manufacturer, etc. [12].

\section{Discussion of results}

A complex technological device, such as a turbine unit, consists of a set of elements and assemblies that are interconnected and subjected to vibration loads. From this, it follows that the failure of one of the elements can lead to a malfunction of the entire power machine.

The existing functional dependencies of the TU elements and the multitude of structural parameters make it difficult to describe its behavior. The existing techniques and methods that simplify the process of diagnosing such systems consist of representing the automation object as a set of separate modules, consisting of smaller functional units. In other words, TU is divided into modules, which, in turn, are divided into functional units, resulting in a hierarchical structure. This division of the TU helps to simplify the procedure for finding suspected malfunctions, which subsequently lead to a failure.

The developed block diagrams make it possible to use the open top-level network protocol CANopen for connecting embedded devices in transport and industrial networks. Such an approach allows collecting all information about the object, basic and current indicators of monitored quantities in one device, indicating the direction of searching for possible malfunctions.

\section{Conclusion}

The structure of a microprocessor-based vibration control system, consisting of a network of autonomous units, each of which serves its own functional unit, is most consistent with the functional and technological structure of the object. The system based on a high-performance data transmission network contains a set of autonomous intelligent units. Combining intelligent blocks with each other occurs using interfaces and protocols functioning on their basis 
Thus, we can say with confidence that the use of the CANopen protocol in vibration control systems for rotary machines is a promising direction that will allow engineers to solve the necessary technical problems using modern methods.

\section{References}

1. V.I. Mindrin, G.V. Pachurin, M.N. Rebrushkin, Sovremennye naukoemkie tekhnologii, 5, 32 (2015)

2. Istinnyh vinovnikov nikogda ne nakazhut, https://www.sibreal.org/a/30111114.html

3. A.A. Igolkin, A.I. Safin, G.M. Makar'yanc, A.N. Kryuchkov, E.V. SHahmatov, Prikladnaya fizika, 4, 49 (2013)

4. A.S. Gol'din Vibraciya rotornyh mashin (M.: Mashinostroenie, 1999)

5. D.A. Plotnikov, Izvestiya vuzov. Elektromekhanika, 3, 96 (2000)

6. V.I. Lachin, Informacionnye tekhnologii i upravlenie: yubilejnyj sb. nauch. tr. fakul'teta informacionnyh tekhnologij i upravleniya, 69 (2001)

7. D.A. Plotnikov, Komp'yuternye tekhnologii $\mathrm{v}$ nauke, proizvodstve, social'nyh i ekonomicheskih processah: materialy XI Mezhdunar. nauch.-prakt. Konf, 19 (2010)

8. V.V. Petruhin, S.V. Petruhin, Osnovy vibrodiagnostiki i sredstva izmereniya vibracii (Infra Inzheneriya, 2010)

9. CANopen User Guide, https://www.logiccontrol.com/datasheets/12/Manuals/CANopen/User\%20Guide/CANopen\%20User\%20 Guide.pdf

10. Vvedenie v protokol CAN, http://can.marathon.ru/page/can-protocols/canbus/canintro

11. Protokol CANopen. Dal'nejshee razvitie CAN. Elektronika NTB, 3 (2006)

12. Ispol'zovanie seti CAN i steka CANopen, https://habr.com/ru/post/144435/ 